\title{
The epidemiology and importance of vitamin B12 screening in diabetic patients
}

\author{
Abrar A. Al Yamani ${ }^{1 *}$, Jameel A. Awadain², Yousif A. Al Saleh ${ }^{3}$, Mohammad S. Baothman ${ }^{4}$, \\ Feras H. Alhussainy ${ }^{5}$, Mazen S. Alshehri ${ }^{6}$, Helal J. Alharthi ${ }^{7}$, Abdulmalek R. Alqwizani ${ }^{8}$, \\ Abdulrahman A. Alrehaili ${ }^{9}$, Ruwaidah H. Melebary ${ }^{10}$, Manar H. Alanazi ${ }^{11}$
}

\author{
${ }^{1}$ Obhur Primary Health Care Center, King Abdullah Medical Complex, Jeddah, Saudi Arabia \\ ${ }^{2}$ Department of Nephrology, ${ }^{6}$ Department of Pharmacy, Al Noor Specialist Hospital, Mecca, Saudi Arabia \\ ${ }^{3}$ College of Medicine, King Faisal University, Al Ahsa, Saudi Arabia \\ ${ }^{4}$ Primary Healthcare Center, East Jeddah Hospital, Jeddah, Saudi Arabia \\ ${ }^{5}$ Abha Primary Healthcare Center, Ministry of Health, Abha, Saudi Arabia \\ ${ }^{7}$ Department of Internal Medicine, King Faisal Complex, Taif, Saudi Arabia \\ ${ }^{8}$ Public Health, ${ }^{9}$ Primary Healthcare Center, Ministry of Health, Medina, Saudi Arabia \\ ${ }^{10}$ College of Medicine, Umm Al-Qura University, Mecca, Saudi Arabia \\ ${ }^{11}$ College of Medicine, Northern Border University, Arar, Saudi Arabia
}

Received: 13 December 2021

Accepted: 15 December 2021

*Correspondence:

Dr. Abrar A. A. Yamani,

E-mail: abrar.yamani2@gmail.com

Copyright: (C) the author(s), publisher and licensee Medip Academy. This is an open-access article distributed under the terms of the Creative Commons Attribution Non-Commercial License, which permits unrestricted non-commercial use, distribution, and reproduction in any medium, provided the original work is properly cited.

\begin{abstract}
Peripheral neuropathy is a commonly reported chronic adverse event among diabetes mellitus (DM) patients secondary to poor glycemic control. It might also result secondary to deficiency of vitamin B12, reportedly common among diabetic patients. Deficiency of vitamin B12 might result from prolonged metformin administration in patients with type II DM (T2DM). It might also result from reduced absorption and impaired metabolism-related events in type I DM (T1DM) patients. This occurs secondary to the presence of associated autoimmune disorders. Vitamin B12 deficiency is a commonly encountered condition among diabetic patients, both T1DM and T2DM, with variable etiologies. Our current study discussed the epidemiology and importance of screening of vitamin B12 in these patients. However, our findings show that screening is not commonly practiced in different settings. Therefore, awareness is low about the benefits and complications of this practice. Therefore, further research is encouraged to alleviate the quality of care in diabetic patients. Screening for vitamin B12 deficiency might intervene against any potential complications, including irreversible, painful, and potentially disabling nerve injury. Accordingly, it is recommended that screening should be initiated since the start of metformin administration and every year or when relevant clinical manifestations were reported.
\end{abstract}

Keywords: Anemia, Vitamin B12 deficiency, Diabetes mellitus, Epidemiology, Management, Screening

\section{INTRODUCTION}

Diabetes mellitus (DM) is a common endocrine disorder that is highly prevalent across different global countries. The disease is correlated with a high mortality and morbidity rate that significantly reduce the quality of life of the impacted patients. ${ }^{1}$ Controlling blood glucose levels is challenging for many patients because of reduced compliance with the proposed restricting management approaches. Furthermore, management of the disease requires integrating other approaches to managing various associated complications with the condition. ${ }^{2,3}$ 
Evidence shows that many complications can develop among diabetic patients, probably due to poor glycemic control and medication administration. Some of the reported complications include cardiovascular-related complications and other events as peripheral neuropathy. ${ }^{4,5}$ Peripheral neuropathy might also result secondary to deficiency of vitamin B12, reportedly common among diabetic patients. Deficiency vitamin B12 might result from prolonged metformin administration in patients with type II DM (T2DM). It might also result from reduced absorption and impaired metabolism-related events in type I DM (T1DM) patients. This occurs secondary to the presence of associated autoimmune disorders. ${ }^{6}$ Previous studies have reported that screening vitamin B12 among diabetic patients would be vital to achieving early intervention against vitamin B12 deficiency and subsequent complications..$^{7-9}$ The present literature review aims to shed more light on The epidemiology and importance of vitamin B12 screening in patients with diabetes.

\section{METHODS}

This literature review is based on an extensive literature search in Medline, Cochrane, and EMBASE databases which was performed on 27 November 2021 using the medical subject headings (MeSH) or a combination of all possible related terms, according to the database. To avoid missing potential studies, a further manual search for papers was done through Google Scholar while the reference lists of the initially included papers. Papers discussing the epidemiology and importance of vitamin B12 screening in diabetic patients were screened for useful information. No limitations were posed on date, language, age of participants, or publication type.

\section{DISCUSSION}

Many previous studies have indicated that T1DM and T2DM patients are at increased risk of having a deficiency of vitamin B12. ${ }^{10-14}$ Among the different investigations of T2DM, it has been shown that frequently using metformin is the major etiology of developing deficiency of vitamin B12. ${ }^{15,16}$ The studies that reported that metformin is the major reason for developing deficiency of vitamin B12 among diabetic patients estimated that the frequency of this event ranges between $5.8-33 \% .^{13-15}$ The reported different prevalence rates might be attributed to the different measurements of deficiency of vitamin B12 across the relevant investigations. Furthermore, evidence shows that comparing the public and patients with diabetes is complex because definitions of deficiency of vitamin B12 are hugely variable, and the different demographics and behavioral and cultural beliefs. For example, a previous population-based study in Finnland demonstrated that the frequency of deficiency of vitamin B12 was $12.1 \%$ between elderly diabetics (65-100 years old). Moreover, it has been demonstrated that deficiency of vitamin B12 was previously diagnosed in $2.6 \%$ of the included patients. The exact rate was also estimated for having vitamin B12 replacement therapy. ${ }^{17}$ Religious and cultural beliefs are also important to consider when assessing and evaluating the dificiency of vitamin B12. For instance, previous research from India, where a big part of the population is vegetarian because of their religious beliefs, demonstrated that around two-thirds $(67 \%)$ of the included participants had been diagnosed with a deficiency of vitamin B12. This was furtherly evidenced that reduced frequency of deficiency of vitamin B12 was remarkably associated with a vegetarian diet when multivariate analysis. ${ }^{18}$ However, it should be noted that other investigations in India also demonstrated lower prevalence rates among their populations. For instance, Shobha et al. reported that the estimated frequency of deficiency of vitamin B12 in their study was $16 \%$ among <60-year-old participants. However, it has been shown that the frequency significantly increased to $55 \%$ when the authors used serum methylmalonic acid to assess vitamin B12 deficiency, being a more sensitive tool. ${ }^{19}$

Together with lifestyle modifications, metformin has been suggested as the primary line of therapy for T2DM due to its favorable effects on reducing blood glucose levels. Moreover, it has been reported to have lower hepatic and renal complications. ${ }^{20,21}$ However, despite all of the reported favorable events, it has been indicated that vitamin B12 deficiency has been associated with the longterm administration of metformin. Therefore, DeFronzo et al conducted a previous randomized controlled trial. ${ }^{22}$ They found that vitamin B12 reduction was significantly reported among patients with metformin administration by $29 \%$ and $22 \%$ more than patients with glyburide and placebo administration. Similar findings were also reported among various investigations in the literature, including randomized controlled trials, cross-sectional studies, and single case reports. ${ }^{10-13,16,23}$

It should be noted that different factors usually contribute to the effect of metformin on vitamin B12 deficiency. Some of these factors include the duration of administration, dose of metformin, and patient age. ${ }^{23-26}$ This has been furtherly indicated in a previous case-control study in China. The authors found that an odds ratio of 2.9 for vitamin B12 deficiency was associated with one g/day increase in the dose of metformin administration. It has been further estimated that an odds ratio of 2.4 for developing vitamin B12 deficiency for patients using metformin for $\geq 3$ years compared to other patients who administered the drug for a shorter duration. ${ }^{24}$ A previous investigation also reported that the negative effect of metformin over reducing vitamin B12 absorption usually takes place since the $4^{\text {th }}$ month after administration. ${ }^{27}$ However, another study reported that the clinical manifestation is not usually exhibited until 5-10 years after administration because of the high liver stores of vitamin B12. ${ }^{28}$ In addition, different actions were reported for metformin, which reduces the serum levels of vitamin B12. Some of these factors include interacting with the cubulin endocytic receptor, altering levels of intrinsic factor, and inactivation and competitive inhibition of vitamin B12 
absorption. Other factors include vitamin B12 secondary to bacterial overgrowth and inhibition of calcium dependant vitamin B12-intrinsic factor absorption at the distal ileum. ${ }^{28-30}$

The epidemiology of vitamin B12 screening and deficiency was also reported among patients with T1DM. However, the main reason for developing these events is probably the development of related autoimmune disorders, common among patients with T1DM. For instance, evidence shows that pernicious anemia is a potential factor for vitamin B12 deficiency. Thus, the disease is an autoimmune disorder that is more prevalent among patients with T1DM than in the general population. ${ }^{31,32}$ A previous study in India reported that the prevalence of vitamin B12 deficiency among patients with T1DM ranged between $45.5 \%$ and $54 \%$ based on the definition of vitamin B12 deficiency. However, it should be noted that the authors reported that vitamin B12 deficiency was not significantly correlated with the level of glycemic control, duration of DM, age, and gender. ${ }^{33}$ In addition, many previous studies have reported the presence of specific antibodies that might contribute to the pathology of vitamin B12 deficiency among patients with T1DM. Some of the reported antibodies include parietal cell antibodies, autoantibodies against intrinsic factor types I and II. This is particularly common among patients with HLA-DQA $1 * 0501-\mathrm{B} 1 * 0301$ haplotype and glutamate decarboxylase- 65 antibodies. These antibodies contribute to the development of pernicious anemia among patients with T1DM more than the general population. This significantly leads to reduced vitamin B12 and secondary deficiency. ${ }^{31,34-36}$ It has been furtherly demonstrated that the metabolism of vitamin B12 is also impacted by celiac disease and primary autoimmune hypothyroidism. These conditions are reportedly common among patients with T1DM. ${ }^{37-40}$ For instance, a previous cross-sectional investigation estimated that primary autoimmune hypothyroidism and celiac disease prevalence was $0.6 \%$ and $20.2 \%$ among patients with T1DM. ${ }^{41}$ Bacterial overgrowth, bowel wall edema, decreased bowel motility, and reduced absorption is all factors that contribute to vitamin B12 deficiency secondary to autoimmune thyroiditis. Other factors also include dyserythropoietic, reduced oral intake, and the presence of autoantibodies against intrinsic factors and parietal cells secondary to reduced thyroid hormones. ${ }^{37}$ The epidemiology of vitamin B12 deficiency was also reported among other investigations. It has been demonstrated that celiac disease is prevalent among $16 \%$ of T1DM patients. ${ }^{38}$ Associated malabsorption is the main factor contributing to developing vitamin B12 deficiency among these patients. ${ }^{42}$

Recent evidence shows that patients with metformin medication should have routine screening against vitamin B12 deficiency to intervene against the development of serious nerve damage. In addition, it has been shown that early detection of the condition might provide good prevention against the development of a potentiallydisabling, painful, and irreversible nerve injury. However, previous studies demonstrated that many diabetic patients have vitamin B12 deficiency secondary to metformin use, and the association is dose and duration dependant. Therefore, healthcare authorities should exert more efforts in screening and early identification of diabetic patients with vitamin B12 deficiency to achieve optimal management and reduce adverse events. ${ }^{43-45}$ In addition, healthcare authorities should also target increasing knowledge about patients to enhance attitudes and practice care towards administering metformin. However, according to relevant research, not many institutions perform routine screening. Therefore, not many healthcare professionals and patients are aware of the vitality of this step in managing vitamin B12 deficiency. ${ }^{46}$

Evidence shows no current guidelines about the favorable screening events for vitamin B12 deficiency among T1DM and T2DM patients. This is probably secondary to reducing adequate investigations in the literature elaborating the effectiveness of such screening approaches for the targeted population. However, it should be recommended that clinicians should advise their T2DM patients to perform routine screening of vitamin B12 before initiating metformin administration. In the same context, routine annual screening of vitamin B12 should also be indicated for elderly patients using metformin for T2DM, that have clinically worsening manifestations of polyneuropathy, that administer high doses of metformin ( $\geq 2 \mathrm{~g} /$ day), and that administer metformin for a prolonged period ( $\geq 3-4$ years). It should be noted that the evidence suggests that routine screening should be specific for these criteria, irrespective of the presence of any other hematological abnormalities. ${ }^{47}$ Evidence shows that screening of vitamin B12 deficiency is similar in the general population and when performed for patients with diabetes. For patients with T2DM, targeting serum levels of vitamin B12 should be considered the preliminary step in the screening approach for vitamin B12 deficiency in these patients. It has been shown that the diagnosis of vitamin B12 deficiency should be established at a level of $<200 \mathrm{pg} / \mathrm{ml}$. On the other hand, no diagnosis of vitamin B12 deficiency should be considered with a vitamin B12 of $>400 \mathrm{pg} / \mathrm{ml}^{48}$ It is worth mentioning that measuring serum methylmalonic acid and homocysteine are more specific and sensitive for screening and detecting vitamin B12 deficiency. This approach is best recommended for T2DM patients with baseline serum levels of vitamin B12 of 200-400 pg/ml and abnormal hematological characteristics. The normal range, upon which deficiency of vitamin B12 should be based, includes $<0.28 \mu \mathrm{mol} / 1$ and 5-15 $\mu \mathrm{mol} / \mathrm{l}$ for serum methylmalonic acid and homocysteine, respectively. ${ }^{47,49}$ Screening of vitamin B12 deficiency is also beneficial for patients with T1DM. However, no apparent guidelines were found in the literature about the best practice care and screening of vitamin B12 deficiency in these patients. On the other hand, a previous study recommended that routine screening be conducted at baseline since an annual routine screening follows diagnosis for three years. It has been further recommended that screening approaches be 
conducted every five years or in the presence and development of clinical manifestations suggestive of vitamin B12 deficiency. ${ }^{31}$ The recommended routine screening for patients with T1DM is probably due to the high prevalence of pernicious anemia and other autoimmune diseases in these patients and the subsequent development of vitamin B12 deficiency. Therefore, the screening in these settings should be conducted via assessing vitamin B12 levels in the high-risk population. Moreover, it is recommended to assess the presence of the antibodies above among patients with autoimmune disorders. Evidence shows that an increased risk of developing vitamin B12 deficiency is associated with these autoantibodies among T1DM patients. ${ }^{31,50}$

\section{CONCLUSION}

Vitamin B12 deficiency is a commonly encountered condition among diabetic patients, both T1DM and T2DM, with variable etiologies. Our current study discussed the epidemiology and importance of screening of vitamin B12 in these patients. However, our findings show that screening is not commonly practiced in different settings. Therefore, awareness is low about the benefits and complications of this practice. Therefore, further research is encouraged to alleviate the quality of care in diabetic patients. Screening for vitamin B12 deficiency might intervene against any potential complications, including irreversible, painful, and potentially disabling nerve injury. Accordingly, it is recommended that screening should be initiated since the start of metformin administration and every year or when relevant clinical manifestations were reported.

Funding: No funding sources Conflict of interest: None declared

Ethical approval: Not required

\section{REFERENCES}

1. Mekala KC, Bertoni AG. Epidemiology of diabetes mellitus. In: Transplantation, bioengineering, and regeneration of the endocrine pancreas. Elsevier. 2020:49-58.

2. Reusch JE, Manson JE. Management of Type 2 Diabetes in 2017: Getting to Goal. JAMA. 2017;317(10):1015-6.

3. Imam K. Management and treatment of diabetes mellitus. Adv Exp Med Biol. 2012;771:356-80.

4. Bailes BK. Diabetes mellitus and its chronic complications. AORN J. 2002;76(2):266-76.

5. Papatheodorou K, Banach M, Bekiari E, Rizzo M, Edmonds M. Complications of Diabetes 2017. J Diabetes Res. 2018;2018:3086167.

6. Oh R, Brown DL. Vitamin B12 deficiency. Am Fam Phys. 2003;67(5):979-86.

7. Fogelman Y, Kitai E, Blumberg G, Golan-Cohen A, Rapoport M, Carmeli E. Vitamin B12 screening in metformin-treated diabetics in primary care: were elderly patients less likely to be tested? Aging Clin Exp Res. 2017;29.

8. Albai O, Timar B, Paun DL, Sima A, Roman D, Timar R. Metformin Treatment: A Potential Cause of Megaloblastic Anemia in Patients with Type 2 Diabetes Mellitus. Diabetes, metabolic syndrome and obesity : targets and therapy. 2020;13:3873-8.

9. Owhin SO, Adaja TM, Fasipe OJ, Akhideno PE, Kalejaiye OO, Kehinde MO. Prevalence of vitamin $\mathrm{B}(12)$ deficiency among metformin-treated type 2 diabetic patients in a tertiary institution, South-South Nigeria. SAGE Open Med. 2019;7:2050312119853433.

10. Kumthekar AA, Gidwani HV, Kumthekar AB. Metformin associated B12 deficiency. J Assoc Phys India. 2012;60:58-60.

11. Liu KW, Dai LK, Jean W. Metformin-related vitamin B12 deficiency. Age and ageing. 2006;35(2):200-1.

12. Nervo M, Lubini A, Raimundo FV. Vitamin B12 in metformin-treated diabetic patients: a cross-sectional study in Brazil. Revista da Associacao Medica Brasileira (1992). 2011;57(1):46-9.

13. Pflipsen MC, Oh RC, Saguil A, Seehusen DA, Seaquist D, Topolski R. The prevalence of vitamin $\mathrm{B}(12)$ deficiency in patients with type 2 diabetes: a cross-sectional study. J Am Board Fam Med. 2009;22(5):528-34.

14. Qureshi SA, Ainsworth A, Winocour PH. Metformin therapy and assessment for vitamin B12 deficiency: is it necessary? Practical Diabetes. 2011;28(7):302-4.

15. Reinstatler L, Qi YP, Williamson RS, Garn JV, Oakley GP. Association of biochemical $\mathrm{B}_{12}$ deficiency with metformin therapy and vitamin $\mathrm{B}_{12}$ supplements: the National Health and Nutrition Examination Survey, 1999-2006. Diabetes Care. 2012;35(2):327-33.

16. Kos E, Liszek MJ, Emanuele MA, Durazo-Arvizu R, Camacho P. Effect of metformin therapy on vitamin $\mathrm{D}$ and vitamin $\mathrm{B}_{12}$ levels in patients with type 2 diabetes mellitus. Endocrine Practice. 2012;18(2):179-84.

17. Loikas S, Koskinen $\mathrm{P}$, Irjala K. Vitamin B12 deficiency in the aged: a population-based study. Age and ageing. 2007;36(2):177-83.

18. Yajnik CS, Deshpande SS, Lubree HG, et al. Vitamin B12 deficiency and hyperhomocysteinemia in rural and urban Indians. The Journal of the Association of Physicians of India. 2006;54:775-782.

19. Shobha V, Tarey SD, Singh RG. Vitamin $B_{12}$ deficiency \& levels of metabolites in an apparently normal urban south Indian elderly population. Indian J Med Res. 2011;134(4):432-9.

20. Standards of medical care in diabetes--2012. Diabetes Care. 2012;35(1):11-63.

21. Day C. ADA-EASD diabetes guidance: individualised treatment of hyperglycaemia. $\mathrm{Br} \mathrm{J}$ Diabetes Vascular Dis. 2012;12(3):146-51.

22. DeFronzo RA, Goodman AM. Efficacy of metformin in patients with non-insulin-dependent diabetes 
mellitus. The Multicenter Metformin Study Group. N Engl J Med. 1995;333(9):541-9.

23. de Jager J, Kooy A, Lehert P. Long term treatment with metformin in patients with type 2 diabetes and risk of vitamin B-12 deficiency: randomised placebo controlled trial. BMJ. 2010;340:2181.

24. Ting RZ, Szeto CC, Chan MH, Ma KK, Chow KM. Risk factors of vitamin $\mathrm{B}(12)$ deficiency in patients receiving metformin. Arch Int Med. 2006;166(18):1975-9.

25. Son PT, Reda A, Viet DC. Exchange transfusion in the management of critical pertussis in young infants: a case series. Vox Sang. 2021;116(9):976-82.

26. Dibas M, Doheim MF, Ghozy S, Ros MH, El-Helw GO, Reda A. Incidence and survival rates and trends of skull Base chondrosarcoma: A Population-Based study. Clin Neurol Neurosurg. 2020;198:106153.

27. Wulffelé MG, Kooy A, Lehert P, et al. Effects of short-term treatment with metformin on serum concentrations of homocysteine, folate and vitamin B12 in type 2 diabetes mellitus: a randomized, placebo-controlled trial. Journal of internal medicine. 2003;254(5):455-463.

28. Andrès E, Noel E, Goichot B. Metformin-associated vitamin B12 deficiency. Arch Int Med. 2002;162(19):2251-2.

29. Bauman WA, Shaw S, Jayatilleke E, Spungen AM, Herbert V. Increased intake of calcium reverses vitamin B12 malabsorption induced by metformin. Diabetes Care. 2000;23(9):1227-31.

30. Nguyen TM, Huan VT, Reda A. Clinical features and outcomes of neonatal dengue at the Children's Hospital 1, Ho Chi Minh, Vietnam. J Clin Virol. 2021;138:104758.

31. De Block CE, De Leeuw IH, Van Gaal LF. Autoimmune gastritis in type 1 diabetes: a clinically oriented review. J Clin Endocrinol Metab. 2008;93(2):363-71.

32. Van den Driessche A, Eenkhoorn V, Van Gaal L, De Block C. Type 1 diabetes and autoimmune polyglandular syndrome: a clinical review. Netherlands J Med. 2009;67(11):376-87.

33. Koshy AS, Kumari SJ, Ayyar V, Kumar P. Evaluation of serum vitamin B12 levels in type 1 diabetics attending a tertiary care hospital: A preliminary cross - sectional study. Indian J Endocrinol Metab. 2012;16(1):79-82.

34. De Block CE, De Leeuw IH, Rooman RP, Winnock F, Du Caju MV, Van Gaal LF. Gastric parietal cell antibodies are associated with glutamic acid decarboxylase-65 antibodies and the HLA DQA1*0501-DQB1*0301 haplotype in Type 1 diabetes mellitus. Belgian Diabetes Registry. Diabetic Med. 2000;17(8):618-22.

35. De Block CE, De Leeuw IH, Van Gaal LF. High prevalence of manifestations of gastric autoimmunity in parietal cell antibody-positive type 1 (insulindependent) diabetic patients. The Belgian Diabetes
Registry. J Clin Endocrinol Metab. 1999;84(11):4062-7.

36. Riley WJ, Toskes PP, Maclaren NK, Silverstein JH. Predictive value of gastric parietal cell autoantibodies as a marker for gastric and hematologic abnormalities associated with insulin-dependent diabetes. Diabetes. 1982;31(12):1051-5.

37. Fein HG, Rivlin RS. Anemia in thyroid diseases. Med Clin North Am. 1975;59(5):1133-45.

38. Rewers M, Liu E, Simmons J, Redondo MJ, Hoffenberg EJ. Celiac disease associated with type 1 diabetes mellitus. Endocrinol Metab Clin North Am. 2004;33(1):197-214.

39. Roberts CG, Ladenson PW. Hypothyroidism. Lancet (London, England). 2004;363(9411):793-803.

40. Barker JM. Clinical review: Type 1 diabetesassociated autoimmunity: natural history, genetic associations, and screening. J Clin Endocrinol Metab. 2006;91(4):1210-7.

41. Joffe B, Distiller L, Landau S, Blacking L, Klisiewicz A. Spectrum of Autoimmune Disorders in Type 1 Diabetes-A Cross-Sectional Clinical Audit. J Diabetes Metab. 2010;1(112):2.

42. Selimoğlu MA, Karabiber H. Celiac disease: prevention and treatment. J Clin Gastroenterol. 2010;44(1):4-8.

43. Kim J, Ahn CW, Fang S, Lee HS, Park JS. Association between metformin dose and vitamin B12 deficiency in patients with type 2 diabetes. Medicine (Baltimore). 2019;98(46):e17918.

44. Ko SH, Ko SH, Ahn YB. Association of vitamin B12 deficiency and metformin use in patients with type 2 diabetes. J Korean Med Sci. 2014;29(7):965-72.

45. Sato Y, Ouchi K, Funase Y, Yamauchi K, Aizawa T. Relationship between metformin use, vitamin B12 deficiency, hyperhomocysteinemia and vascular complications in patients with type 2 diabetes. Endocrine J. 2013;60(12):1275-80.

46. Alshammari AN, Iqbal R, Baksh IP. Vitamin B12 deficiency and the knowledge and practice of physicians regarding screening for vitamin B12 deficiency among type 2 diabetic patients on metformin in selected hospitals in Riyadh, Saudi Arabia. J Family Med Prim Care. 2019;8(7):2306-11.

47. Mazokopakis EE, Starakis IK. Recommendations for diagnosis and management of metformin-induced vitamin B12 (Cbl) deficiency. Diabetes Res Clin Pract. 2012;97(3):359-67.

48. Snow CF. Laboratory diagnosis of vitamin B12 and folate deficiency: a guide for the primary care physician. Arch Int Med. 1999;159(12):1289-98.

49. Klee GG. Cobalamin and folate evaluation: measurement of methylmalonic acid and homocysteine vs vitamin $\mathrm{B}(12)$ and folate. Clin Chem. 2000;46(8):1277-83.

Cite this article as: Yamani AAA, Awadain JA, Saleh YAA, Baothman MS, Alhussainy FH, Alshehri MS, et al. The epidemiology and importance of vitamin B12 screening in diabetic patients. Int $\mathbf{J}$ Community Med Public Health 2022;9:471-5. 